\title{
ChemComm
}

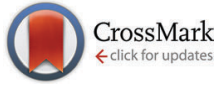

Cite this: Chem. Commun., 2014, 50, 15427

Received 9th July 2014

Accepted 13th October 2014

DOI: $10.1039 / \mathrm{c} 4 \mathrm{cc} 04901 \mathrm{~b}$

www.rsc.org/chemcomm

\section{Compartmentalization of bacteria in microcapsules $\dagger$}

\author{
Judith van Wijk, ${ }^{a}$ Tiaan Heunis, ${ }^{b}$ Elrika Harmzen, ${ }^{c}$ Leon M. T. Dicks, ${ }^{b}$ Jan Meuldijk ${ }^{a}$ \\ and Bert Klumperman*c
}

\begin{abstract}
Lactobacillus plantarum strain $\mathbf{4 2 3}$ was encapsulated in hollow poly(organosiloxane) microcapsules by templating water-in-oil Pickering emulsion droplets via the interfacial reaction of alkylchlorosilanes. The bacteria were suspended in growth medium or buffer to protect the cells against $\mathrm{pH}$ changes during the interfacial reactions with alkylchlorosilanes. The results of this work open up novel avenues for the encapsulation of microbial cells.
\end{abstract}

Encapsulation of viable microbial cells has several novel applications in the pharmaceutical, food and agricultural industries. ${ }^{1-3}$ Critical for these potential applications is that the microcapsules are permeable to small molecules and even macromolecules, but impermeable to the encapsulated microbial cells. It is also a prerequisite that the encapsulated cells remain viable during and after encapsulation. This is challenging, as most microorganisms are sensitive to changes in the environmental conditions, such as temperature, $\mathrm{pH}$ and the presence of cytotoxic chemicals. Viable cells have previously been encapsulated in gels, often in the form of microbeads. These include hydrogels, ${ }^{4,5}$ calcium alginate $^{6}$ and sol-gel products. ${ }^{7-9}$ Hollow calcium carbonate capsules produced using a layer-by-layer technique have been used to encapsulate individual E. coli cells. ${ }^{10}$ These methods trap the bacteria in a matrix or just encapsulate one individual bacterium. In contrast, in the present work, multiple bacteria suspended in Tris-HCl buffer ( $\mathrm{pH}$ 8.0) are encapsulated in microcapsules. Here we report on an encapsulation method, which results in minimum contamination of the dispersed phase, causing bacteria to remain viable inside the microdroplets. Contamination caused by, e.g. a pH change, would result in cell death. Contamination that causes a change in $\mathrm{pH}$ should thus not exceed the buffer

\footnotetext{
${ }^{a}$ Eindhoven University of Technology, Department of Chemical Engineering and Chemistry, P.O. Box 513, 5600 MB Eindhoven University of Technology, The Netherlands

${ }^{b}$ Department of Microbiology, Stellenbosch University, Private Bag X1, 7602 Matieland, South Africa

${ }^{c}$ Department of Chemistry and Polymer Science, Stellenbosch University, Private Bag X1, Matieland 7602, South Africa. E-mail: bklump@sun.ac.za $\dagger$ Electronic supplementary information (ESI) available: Experimental procedures and some supporting figures. See DOI: 10.1039/c4cc04901b
}

capacity. The beneficial characteristics of sol-gel silica as an encapsulation material include the controllable porosity and mechanical properties of the shell. These characteristics can be controlled by the selection of precursors, modification agents and synthesis conditions. ${ }^{11}$ Pickering emulsion droplets are known to be suitable templates for microcapsules because of their high colloidal stability and their controllable droplet size. ${ }^{12-14}$ This communication reports on the encapsulation of L. plantarum in hollow poly(organosiloxane) microcapsules. To the authors' knowledge, this is the first time that viable bacteria have been encapsulated in microcapsules templated on a Pickering emulsion. These microcapsules are synthesized from water-in-oil emulsions stabilized by silica microparticles by an interfacial reaction of alkylchlorosilanes, see Fig. 1A. The alkylchlorosilanes have hydrophobic properties, i.e. they are not miscible with water before and after hydrolysis. Alkylchlorosilanes are very reactive towards water and contribute to the stabilization of the water droplets by the microparticles. Hence, the monomer is oil-soluble and rapidly polymerizes upon contact with the stabilizing particles or with water at the interface of the droplets. The viability of the
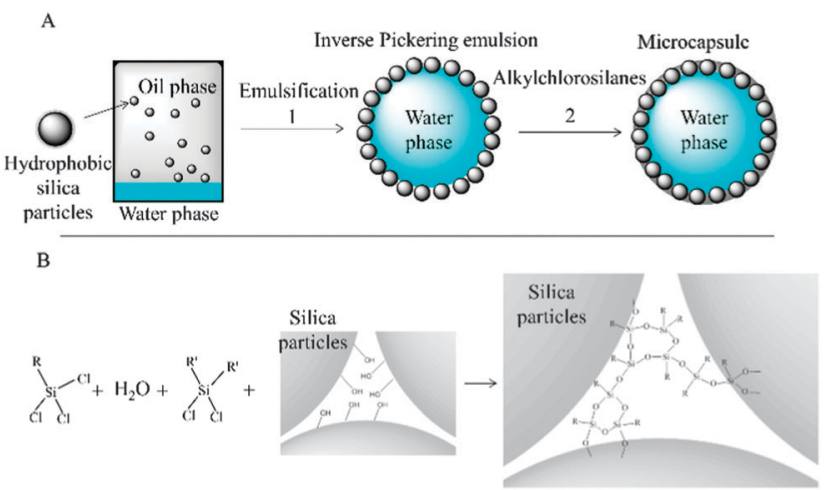

Fig. 1 Methodology for the synthesis of microcapsules. (A) An inverse Pickering emulsion is produced by the emulsification of water in a dispersion of hydrophobic silica particles in $n$-heptane (1). The addition of alkylchlorosilanes to the inverse Pickering emulsion results in an interfacial reaction with water to produce polymer, thus forming microcapsules (2). (B) The reactive silanol groups on the primary stabilizing silica particles will also react with the poly(alkyl siloxane). 
bacteria was determined in the Pickering emulsion droplets and in the microcapsules by using confocal fluorescence microscopy.

The method used to prepare microcapsules is schematically presented in Fig. 1. The objective of this work was to encapsulate viable bacteria and as a consequence, a water-in-oil (or inverse) Pickering emulsion was required, since bacteria generally do not survive in organic media, with only very few exceptions. ${ }^{15}$ The threephase contact angle that the particles have at the oil-water interface, when used in a Pickering emulsion, is a strong indication of the stability of the emulsion. ${ }^{16}$ The most stable inverse Pickering emulsions are produced when the stabilizing particles have a threephase contact angle between $94^{\circ}$ and $110^{\circ}$, meaning the particles are slightly hydrophobic. ${ }^{17}$ Silica microparticles that are modified by alkylsilanes are known to have a three-phase contact angle suitable for inverse Pickering stabilization. ${ }^{18}$ Water was added to the dispersion of modified particles in heptane to form the inverse Pickering emulsion (Fig. 1). The water, oil and particle concentrations in the emulsion were calculated according to Salari et al. ${ }^{19}$ and the target droplet radius was always set at $25 \mu \mathrm{m}$, unless indicated otherwise. The Pickering emulsion was produced manually, by shaking the mixture of water, $n$-heptane and the hydrophobic $\mathrm{SiO}_{2}$ particles for 30 seconds. The reason for choosing this methodology is that bacteria are not able to survive high shear or ultra-sonication, which is usually applied to effect emulsification. Alkylchlorosilanes were added to the inverse Pickering emulsion that reacted with water at the interface (Fig. 1B). The reaction of these alkylchlorosilanes proceeds first by a rapid hydrolysis of the monomer with water, producing a surfactant-resembling monomer (eqn (1)). ${ }^{20}$ The hydrolysis is followed by the condensation reactions (eqn (2)), producing the shell around the microemulsion droplets, as depicted in Fig. 1B.

$$
\begin{aligned}
\mathrm{RSiCl}_{3}(\mathrm{liq})+\mathrm{H}_{2} \mathrm{O} \rightarrow & \mathrm{RSi}(\mathrm{OH})_{3} \text { (interface) } \\
& +\mathrm{HCl}(\mathrm{aq}) \text { hydrolysis reaction }
\end{aligned}
$$

$2 \mathrm{RSi}(\mathrm{OH})_{3}$ (interface) $\rightarrow \mathrm{R}(\mathrm{OH})_{2} \mathrm{SiOSi}(\mathrm{OH})_{2} \mathrm{R}$ (interface)

$$
+\mathrm{H}_{2} \mathrm{O} \text { condensation reaction }
$$

The particles that stabilize the inverse Pickering emulsion were produced using the Stöber method. ${ }^{21}$ These silica particles were used in a seeded silica polymerization to produce monodisperse particles in the micron-size range. ${ }^{22}$ After centrifugation, the particles were air-dried and re-dispersed in $n$-heptane. Subsequently, the $\mathrm{SiO}_{2}$ particles obtained were modified with 3-(trimethoxysilyl)-propyl methacrylate (MPTS) in $n$-heptane to give them a hydrophobic character, ${ }^{18}$ see ESI, $\dagger$ eqn (S2).

The average concentration of reactive silanol groups on silica particles that are produced using the Stöber method is equal to $8 \mu \mathrm{mol} \mathrm{m}_{\text {particle }}{ }^{-2} \cdot{ }^{23}$ Besides reactive silanol groups, physically adsorbed water is also present at the surface of the silica microparticles. Amorphous silica is well known to physically adsorb water and the adsorbed water will also react with the modification agent. ${ }^{24}$ Hence, it is assumed that after the modification step, the surface of the silica microparticles is covered with a layer of MPTS. This again resulted in residual reactive hydroxy groups from the MPTS. Fig. 2A is a scanning electron microscopy (SEM) image of silica microparticles that were produced using the Stöber method, followed by

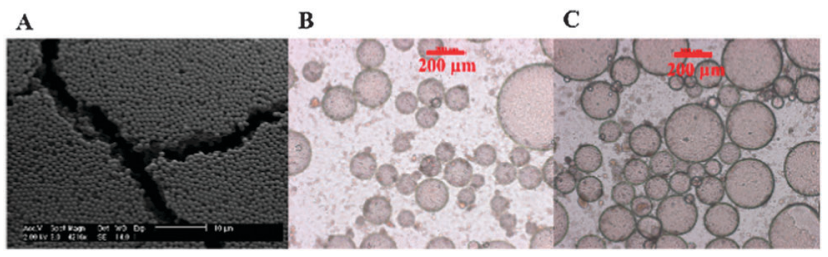

Fig. 2 SEM image of Stöber silica microparticles (A) and light microscopy images of inverse Pickering emulsion droplets stabilized by hydrophobized silica microparticles (B and C).

a seeded polymerization technique. Before modification, the surface area of the microparticles was calculated by using the freeware package Image J. ${ }^{25}$ Water was added to the particle dispersion after modification, followed by manual vigorous shaking of the mixture to produce a stable inverse Pickering emulsion (Fig. 2B and C).

When the microparticles used to stabilize the Pickering emulsion are monodisperse and have the proper three-phase contact angle, they arrange themselves in a hexagonal close packing on the wateroil interface. For this reason Pickering emulsions with a narrow size distribution can be produced with a distribution of $D_{\mathrm{z}} / D_{\mathrm{v}} \approx 1.1$. $^{13}$ However, it takes a certain amount of energy to transfer a particle to the interface and a specific time to bring them all to the interface. When emulsification takes place manually, the amount of energy and the dispersion time are not sufficient to create a Pickering emulsion with narrow size distribution, which is clear from the estimated polydispersity index $D_{\mathrm{z}} / D_{\mathrm{v}} \approx 5.3$ based on Fig. $2 \mathrm{~B}$ and $\mathrm{C}$. Despite the broad droplet size distribution, the emulsions were stable and macroscopic phase separation was not observed over the course of 7 days.

Alkylchlorosilanes are frequently used for the modification of silica surfaces to improve dispersion properties, or to effect crosslinking for the immobilization of catalysts or biomolecules. ${ }^{26}$ When alkylchlorosilanes are used to modify surfaces in the presence of water, they are known to yield a variety of nanostructures, besides a smooth polymer layer on the relevant surface..$^{27-29}$ The underlying reason for the formation of the nanostructures is the hydrolysis and self-condensation of the alkylchlorosilanes in the presence of water that occurs in parallel to reactions with the surface-bound silanol groups. ${ }^{27}$ The formation of the nanostructures is further dependent on the alkyl chain length of the alkylchlorosilane and the size, shape and concentration of the nanostructures are equally dependent on the chain length. ${ }^{27}$

Reaction of the alkylchlorosilanes with the silanol groups on the particles and the network formation between the silica particles should be sufficient to produce a stable microcapsule when a solid shell around an inverse Pickering emulsion is produced. Hydrolysis of a chlorosilane leads to a hydrophilic intermediate due to the produced hydroxy groups (see ESI, $\dagger$ eqn (S2)). It is important that the intermediates do not migrate into the emulsion droplets, but stay at the interface. To restrict the reaction to the interface and to avoid migration into the emulsion droplet, octadecyltrichlorosilane (OTC) was used. To stimulate complete network formation, dimethyldichlorosilane (DMDCS) was used, which is more reactive, but also partially hydrophobic after hydrolysis. After a stable Pickering emulsion was produced, a solution of OTC and DMDCS in $n$-heptane was added (Fig. 1). Silica-based condensation products that have not 


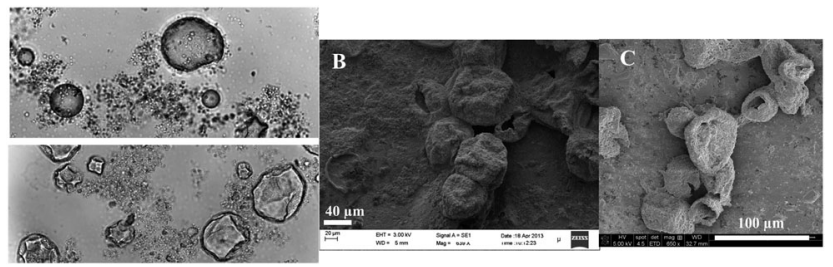

Fig. 3 Light microscopy ( $A$ ) and SEM (B and C) images of the produced microcapsules. Microcapsules were synthesized by the interfacial reaction of octadecyltrichlorosilane and dimethyldichlorosilane at the interface.

reacted with silica particles or with the newly formed silica shell are hydrophobic and will therefore stay in the continuous oil phase.

Light microscopy (LM) and SEM images of microcapsules produced after the addition of OTC and DMDCS to a stable Pickering emulsion is shown in Fig. 3. A polyalkylsiloxane shell is formed around the microdroplets as a result of the interfacial reaction. The reaction of the monomers at the interface with each other, and with the silanol groups at the surface of the particles, also results in the detachment of the particle from the interface because of their increased hydrophobicity (Fig. 3A). Nevertheless, this particle detachment did not destabilize the emulsion to such an extent that macroscopic phase separation took place, before encapsulation. The newly produced polymer surrounding the initially stabilizing silica microparticles can be clearly distinguished when focusing on the shell (Fig. S1F, ESI $\dagger$ ). In contrast to the inverse Pickering emulsion droplets, the microcapsules do not appear to be perfectly spherical (Fig. 3). Deformation is due to the low glass transition temperature $\left(T_{\mathrm{g}}\right)$ of the polymer of $-95^{\circ} \mathrm{C}$ as determined by differential scanning calorimetry (DSC) analysis, see Fig. S2 and S3 (ESI $\dagger$ ). The capsules need to be dried before SEM analysis, and the analysis itself takes place under high vacuum. Consequently, broken capsules and collapsed capsules were expected as a result of evaporation of water from the capsules, see Fig. 3B and C, and S1 (ESI $\dagger$ ).

Microcapsules and the method used for encapsulation of bacteria need to have certain characteristics. For example, the capsules should provide a favorable environment for the bacteria to ensure survival and, in view of future applications, the capsules should also be permeable to allow transport of molecules into the external environment. The latter could be tested by following the release of fluorescent markers with different sizes from the capsules. ${ }^{30}$ The focus of the current study is to develop a method to encapsulate viable $L$. plantarum 423 cells $^{31}$ and to ensure viability throughout and after encapsulation. An inverse Pickering emulsion was produced by using a L. plantarum 423 suspension in De Man, Rogosa, and Sharpe (MRS) broth as the dispersed phase. The growth medium for the bacteria, besides other ingredients, also contains salts and the surfactant Tween 80 that could have an influence on the different interfacial energies in the system. However, the presence of these ingredients did not influence the three-phase contact angle to such an extent that macroscopic phase separation took place. Classical microbiological techniques, such as plating out to determine colonyforming units (CFUs), could not be used to determine viability, as the bacteria would be exposed to $n$-heptane after destabilization of the droplets. We therefore utilized a staining technique based on fluorescent dyes, to evaluate the viability of $L$. plantarum 423 upon
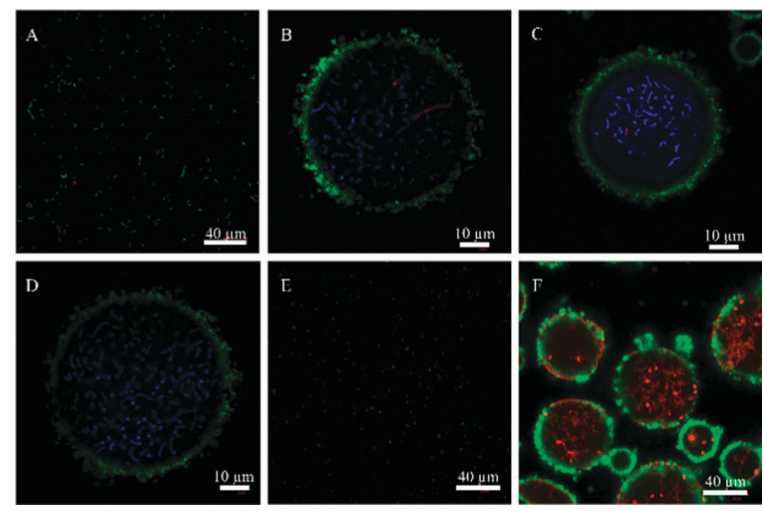

Fig. 4 Inverse Pickering emulsion with L. plantarum 423 imaged by confocal fluorescence microscopy (CFM). (A) Positive control, (B-D) encapsulated L. plantarum 423 in an inverse Pickering emulsion, (E) negative control, (F) negative control for an inverse Pickering emulsion. Blue: DAPI, stains the nucleus of all cells; red: propidium iodide, stains cells with a permeable membrane (non-viable); green: SYTO9, stains the stabilizing silica particles.

dispersion in the droplets of a Pickering emulsion. 4',6-Diamidino-2phenylindole (DAPI, blue) and bis-benzimide trihydrochloride (SYTO 9, green) were added, which should have stained the bacteria, although, interestingly, SYTO9 only stained the silica particles (Fig. 4). In addition, propidium iodide (red) was used, which can only penetrate a cell when the cell membrane is compromised, which is indicative of non-viable bacteria (Fig. 4).

To determine the viability of $L$. plantarum 423 in the emulsion droplets, positive and negative controls were also imaged (Fig. 4A and E). In these control experiments, the bacterial cells were dispersed in a growth medium with SYTO9 (green) and propidium (red dye). Confocal fluorescence microscopy (CFM) was used to image the viable bacteria (Fig. 4A). Non-viable cells were visualized after the addition of ethanol (Fig. 4E). After the addition of $n$-hexylamine to an inverse Pickering emulsion, all bacteria inside the microdroplets died. This image served as a negative control for bacteria in emulsion droplets (Fig. 4F).

When comparing L. plantarum 423, dispersed in MRS broth and emulsified in the Pickering emulsion droplets with the different control samples, it became apparent that at least $90 \%$ of the bacteria remained viable in the emulsion droplets after the emulsification procedure (Fig. 4B-D). The experiment was repeated three times.

Finally, after a stable inverse Pickering emulsion, containing viable L. plantarum 423 was produced, alkylchlorosilanes were added to create a shell around the droplets for final encapsulation. A $\mathrm{pH}$ buffer was added to the water phase to counteract the $\mathrm{pH}$ change that would otherwise be caused by the formation of hydrochloric acid during the polymerization of alkylchlorosilanes. Fluorescent dyes were added before emulsification and the interfacial reaction (Fig. 5 and S4, ESI $\dagger$ ).

The encapsulation procedure resulted in viable $L$. plantarum 423 cells inside the microcapsules (Fig. 5 and S4, ESI $\dagger$ ). The staining technique used is similar to the one used for the Pickering emulsion, using fluorescent dyes, to evaluate viability of L. plantarum 423. Again DAPI (blue) and SYTO 9 (green) were added, which should stain the bacteria. In addition, propidium iodide (red) was used, which can only penetrate a cell when the 


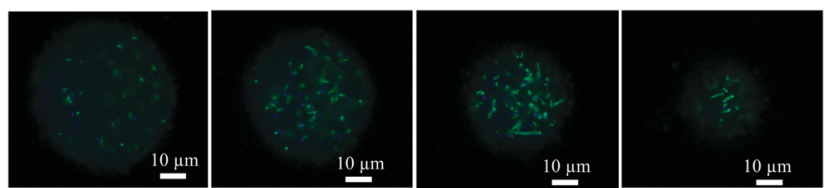

Fig. 5 Confocal Fluorescence Microscopy images (so-called Z-stack) of isolated L. plantarum 423 bacteria in microcapsules. Microcapsules were synthesized by the interfacial reaction of octadecyltrichlorosilane and dimethyldichlorosilane at the interface of inverse Pickering emulsion droplets. Colors are the same as in Fig. 4.

cell membrane is compromised, see Fig. 4E and F. After the interfacial reaction of alkylchlorosilanes, the produced silica capsule wall was not stained by SYTO 9 as in Fig. 4B-D and F. The reason for this different behavior is that the formed polysiloxanes possess very different characteristics from amorphous silica. Again the viable bacteria could be recognized by the blue and green color caused by DAPI and SYTO 9 and the non-viable bacteria by their red color via the addition of propidium iodide. From the CFM images it could be concluded that after the interfacial reaction a large fraction of the bacteria remained viable (Fig. 5 and S4, ESI $\dagger$ ). Fig. 5 is a series of $2 \mathrm{D}$ focus stacking images of an individual capsule. This means that images were made by focusing up and down through the sample/microcapsule. ${ }^{32}$ The images indicate that the bacteria mostly reside at the bottom of the capsules, most likely caused by gravity. In a separate experiment, the viability of the bacteria after the encapsulation process has been monitored. There is no loss of viability within the first three hours after encapsulation (Fig. S5, ESI $\dagger$ ), which means that the encapsulation process and the environment inside the capsule after encapsulation are benign to the bacteria.

In this contribution we have reported the microencapsulation of L. plantarum 423 and showed that the majority of the cells remained viable during and after encapsulation. Encapsulation of L. plantarum 423 was accomplished by the formation of a Pickering emulsion of an aqueous suspension of bacteria in $n$-heptane stabilized by hydrophobized silica particles, followed by the interfacial reaction of alkylchlorosilanes at the interface of inverse the Pickering emulsion droplets that were stabilized by hydrophobized silica microparticles. The bacteria remained viable during and after microcapsule synthesis. This research opens up novel avenues for the encapsulation of bacteria, enzymes and viable cells.

\section{Notes and references}

1 R. Vidhyalakshmi, R. Bhakyaraj and R. S. Subhasree, Encapsulation "The Future of Probiotics"-A Review, Adv. Biol. Res., 2009, 3, 96-103.

2 C. D. Keating, Inorganic Protocells: Gated Access to Microreactors, Nat. Chem., 2013, 5, 449-451.

3 Z. Bu and D. J. E. Callaway, Adv. Protein Chem. Struct. Biol., 2011, 83, 163-221.

4 a. C. Jen, M. C. Wake and a. G. Mikos, Review: Hydrogels for Cell Immobilization, Biotechnol. Bioeng., 1996, 50, 357-364.

5 T. Rossow, J. A. Heyman, A. J. Ehrlicher, A. Langhoff, D. A. Weitz, R. Haag and S. Seiffert, Controlled Synthesis of Cell-Laden Microgels by Radical-Free Gelation in Droplet Microfluidics, J. Am. Chem. Soc., 2012, 134, 4983-4989.

6 S. Sugiura, T. Oda, Y. Izumida, Y. Aoyagi, M. Satake, A. Ochiai, N. Ohkohchi and M. Nakajima, Size Control of Calcium Alginate
Beads Containing Living Cells Using Micro-Nozzle Array, Biomaterials, 2005, 26, 3327-3331.

7 A. Coiffier, T. Coradin, C. Roux, O. M. M. Bouvet and J. Livage, Solgel Encapsulation of Bacteria: A Comparison between Alkoxide and Aqueous Routes, J. Mater. Chem., 2001, 11, 2039-2044.

8 N. Nassif, O. Bouvet, M. Noelle Rager, C. Roux, T. Coradin and J. Livage, Living Bacteria in Silica Gels, Nat. Mater., 2002, 1, 42-44.

$9 \mathrm{~J}$. Livage, T. Coradin and C. Roux, Encapsulation of Biomolecules in Silica Gels, J. Phys.: Condens. Matter, 2001, 673, R673-R691.

10 J. Flemke, M. Maywald and V. Sieber, Encapsulation of Living E. Coli Cells in Hollow Polymer Microspheres of Highly Defined Size, Biomacromolecules, 2013, 14, 207-214.

11 I. Gill, Ballesteros, a. Bioencapsulation within Synthetic Polymers (Part 1): Sol-Gel Encapsulated Biologicals, Trends Biotechnol., 2000, 18, 282-296.

12 S. U. Pickering, Pickering: Emulsions, J. Chem. Soc., 1907, 91, 2001-2021.

13 J. Van Wijk, J. W. O. Salari, N. Zaquen, J. Meuldijk and B. Klumperman, Poly(methyl Methacrylate)-silica Microcapsules Synthesized by Templating Pickering Emulsion Droplets, J. Mater. Chem. B, 2013, 1, 2394-2406.

14 S. A. F. Bon and T. Chen, Pickering Stabilization as a Tool in the Fabrication of Complex Nanopatterned Silica Microcapsules, Langmuir, 2007, 23, 9527-9530.

15 Y. Sardessai and S. Bhosle, Tolerance of Bacteria to Organic Solvents, Res. Microbiol., 2002, 153, 263-268.

16 P. Pieranski, Two-Dimensional Interfacial Colloidal Crystals, Phys. Rev. Lett., 1980, 45, 569-572.

17 G. Kaptay, On the Equation of the Maximum Capillary Pressure Induced by Solid Particles to Stabilize Emulsions and Foams and on the Emulsion Stability Diagrams, Colloids Surf., A, 2006, 282-283, 387-401.

18 S. a. Kulkarni, S. B. Ogale and K. P. Vijayamohanan, Tuning the Hydrophobic Properties of Silica Particles by Surface Silanization Using Mixed Self-Assembled Monolayers, J. Colloid Interface Sci., 2008, 318, 372-379.

19 J. W. O. Salari, G. T. Jemwa, H. M. Wyss and B. Klumperman, Reconstruction of the 3D Structure of Colloidosomes from a Single SEM Image, Soft Matter, 2011, 7, 2033-2041.

20 A. N. Parikh, M. A. Schivley, E. Koo, K. Seshadri, D. Aurentz, K. Mueller, D. L. Allara, V. Pennsyl, S. Uni and U. V. N. Park, Alkylsiloxanes: From Single Monolayers to Layered Crystals. The Formation of Crystalline Polymers from the Hydrolysis of N-Octadecyltrichlorosilane, J. Am. Chem. Soc., 1997, 7863, 3135-3143.

21 W. Stöber, A. Fink and E. Bohn, Controlled Growth of Monodisperse Silica Spheres in the Micron Size Range, J. Colloid Interface Sci., 1968, 69, 62-69.

22 G. Bogush, M. Tracy and C. Z. Iv, Preparation of Monodisperse Silica Particles: Control of Size and Mass Fraction, J. Non-Cryst. Solids, 1988, 104, 95-106.

23 L. T. Zhuravlev, The Surface Chemistry of Amorphous Silica. Zhuravlev Model, Colloids Surf., A, 2000, 173, 1-38.

24 L. T. Zhuravlev, Surface Characterization of Amorphous Silica-a Review of Work from the Former USSR, Colloids Surf., A, 1993, 74, 71-90.

25 M. D. Abràmoff, P. J. Magalhaes and S. Ram, Image Processing with Image, J. Biophotonics, 2004, 1997-2014.

26 Silane coupling agents, connecting across bounderies www.gelest. com (accessed Apr 7, 2014).

27 M. Jin, S. Li, J. Wang, M. Liao and Y. Zhao, Controllable Fabrication of Organosilane Nano-Architectured Surfaces with Tunable Wettability, Appl. Surf. Sci., 2012, 258, 7552-7555.

28 A. Y. Fadeev and T. J. McCarthy, Self-Assembly Is Not the Only Reaction Possible between Alkyltrichlorosilanes and Surfaces: Monomolecular and Oligomeric Covalently Attached Layers of Dichloro- and Trichloroalkylsilanes on Silicon, Langmuir, 2000, 16, 7268-7274.

29 H. S. Khoo and F.-G. Tseng, Engineering the 3D Architecture and Hydrophobicity of Methyltrichlorosilane Nanostructures, Nanotechnology, 2008, 19, 345603.

30 M. P. Neubauer, M. Poehlmann and A. Fery, Microcapsule Mechanics: From Stability to Function, Adv. Colloid Interface Sci., 2014, 207, 65-80.

31 C. A. Van Reenen, L. M. Dicks and M. L. Chikindas, Isolation, Purification and Partial Characterization of Plantaricin 423, a Bacteriocin Produced by Lactobacillus Plantarum, J. Appl. Microbiol., 1998, 84, 1131-1137.

32 LSM 710, LSM 780 Operating Manual, 2010, pp. 134-135. 